\title{
The role of the gut microbiome on the efficacy of immune checkpoint inhibitors in Japanese responder patients with advanced non-small cell lung cancer
}

\author{
Yuki Katayama, Tadaaki Yamada, Takayuki Shimamoto, Masahiro Iwasaku, Yoshiko Kaneko, \\ Junji Uchino, Koichi Takayama \\ Department of Pulmonary Medicine, Graduate School of Medical Science, Kyoto Prefectural University of Medicine, Kyoto, Japan \\ Contributions: (I) Conception and design: Y Katayama, T Yamada; (II) Administrative support: T Yamada, K Takayama; (III) Provision of study \\ materials or patients: T Yamada, T Shimamoto, M Iwasaku, Y Kaneko, J Uchino, K Takayama; (IV) Collection and assembly of data: Y Katayama; (V) \\ Data analysis and interpretation: Y Katayama; (VI) Manuscript writing: All authors; (VII) Final approval of manuscript: All authors. \\ Correspondence to: Tadaaki Yamada, MD, PhD. Department of Pulmonary Medicine, Kyoto Prefectural University of Medicine, 465, Kajii-cho, \\ Kamigyo-ku, Kyoto, 602-8566, Japan. Email: tayamada@koto.kpu-m.ac.jp.
}

\begin{abstract}
Background: Cancer immunotherapy is being developed as a promising alternative for advanced nonsmall cell lung cancer (NSCLC). However, novel biomarkers are required to select patients that will benefit from treatment with immune checkpoint inhibitors (ICIs) for a long period of time. The gut microbiome is expected to be a promising biomarker of ICI response owing to the regulation of the immune status within the host.
\end{abstract}

Methods: In this retrospective study, we included 17 Japanese patients with advanced NSCLC who were treated with ICIs for $>3$ months in our hospital. Fecal samples obtained from the patients during ICI treatment were analyzed by $16 \mathrm{~S}$ ribosomal RNA gene sequencing. We examined the correlation between the diversity of the gut microbiome and treatment with ICIs.

Results: Several bacterial species were more abundant in ICI responders than in non-responders. Patients with abundant Lactobacillus and Clostridium tended to have a longer time to treatment failure (TTF) after receiving ICI than those with a lower abundance.

Conclusions: In conclusion, the composition of the gut microbiome is associated with better clinical benefits from ICI treatment in Japanese patients with NSCLC. A further large-scale study is warranted to validate the composition of the gut microbiome as a novel clinical factor influencing the response to ICIs for an extended time in NSCLC.

Keywords: Immunotherapy; gut microbiome; non-small cell lung cancer (NSCLC); retrospective analysis

Submitted Jul 29, 2019. Accepted for publication Oct 21, 2019.

doi: $10.21037 /$ tlcr.2019.10.23

View this article at: http://dx.doi.org/10.21037/tlcr.2019.10.23

\section{Introduction}

Cancer immunotherapy, such as programmed cell death protein 1 (PD-1)/programmed death-ligand 1 (PD-L1) checkpoint inhibitors, are currently being developed as a promising alternative strategy for the treatment of advanced non-small cell lung cancer (NSCLC) (1-4). Several mechanisms favoring the response to immune checkpoints have been reported, such as a high expression of PD-L1 (5).
However, potent biomarkers that select candidates to have a favorable clinical response to immune checkpoint inhibitors (ICIs) have not been developed.

The gut microbiota, which forms a complex interaction with the human host through immunity and metabolism to maintain homeostasis, consists of 1,000 bacterial species and more than 100 trillion enteric bacteria $(6,7)$. Recent clinical trials have shown the relationship between the 
effects of an anti-PD-1 antibody and the composition of the gut microbiome in malignant melanoma and NSCLC (8-11). Therefore, the gut microbiome has pivotal roles in the prediction of response to ICI treatment in NSCLC patients. In this study, we retrospectively analyzed the relationship between the efficacy of ICI treatment and the gut microbiome in Japanese patients with NSCLC who were administered ICI.

\section{Methods}

\section{Patients}

This was a retrospective study evaluating the effects of gut microbiota on the efficacy of ICIs in long-term responders with advanced NSCLC. We consecutively selected 17 Japanese patients with advanced NSCLC who were treated with ICIs for $>3$ months at the University Hospital Kyoto Prefectural University of Medicine in Japan between December 2015 and March 2018, regardless of any previous treatment with cytotoxic chemotherapy. We collected stool samples one time per patient during ICI treatment between June 2017 and March 2018. We defined responder (R) (partial response to ICI treatment) or non-responder (NR) (stable or progressive disease after ICI treatment at the time of first clinical evaluation) according to the RECIST 1.1 evaluation. Radiological evaluation occurred at least every 12 weeks. The study protocol was approved by the Ethics Committees of Kyoto Prefectural University of Medicine (No. ERB-C-1126), and conducted in accordance with the principles of the Declaration of Helsinki. The TNM stage was classified using version 7 of the TNM stage classification system. Time to treatment failure (TTF) was defined as the time from the start of treatment to treatment discontinuation for any reason. Overall survival (OS) was defined as the treatment start time to death from any cause.

\section{Tumor PD-L1 analysis}

PD-L1 expression was analyzed at SRL, Inc., with the PD-L1 IHC 22C3 pharmDx assay (Agilent Technologies, Santa Clara, CA). The PD-L1 TPS (Tumor Proportion Score) was calculated as the percentage of at least 100 viable tumor cells for complete or partial membrane staining. The pathologists at the commercial vendor interpreted the TPS results.

\section{Samples, DNA extraction, 16S rRNA gene amplification, sequencing, and data analysis}

The gut microbiome analysis was carried out by MykinsoPro gut microbiome testing (Cykinso, Inc., Tokyo, Japan). DNA extraction from fecal samples was performed by an automated DNA extraction machine (GENE PREP STAR PI-480, Kurabo Industries Ltd, Osaka, Japan) according to the manufacturer's protocol. The V1-V2 region of the $16 \mathrm{~S}$ rRNA gene was amplified by RT-PCR. To sequence the $16 \mathrm{~S}$ amplicons by Illumina MiSeq platform, dual index adapters were attached using the Nextera $\mathrm{XT}$ Index kit. The sequence library preparations were performed according to the $16 \mathrm{~S}$ library preparation protocol by Illumina (San Diego, CA). The libraries were sequenced using the MiSeq Reagent Kit v2 (500 Cycles, 250 bp pairedend). After clustering with 97\% homology using UCLUST version 1.2.22q (Edgar, 2010), operational taxonomic units (OTUs) were generated. The representative sequences of the OTUs were collated against the full-length $16 \mathrm{~S}$ gene database Greengenes (v13.8) to determine the identity and composition of the bacterial genera. The QIIME (v1.8.0) pipeline was used for analysis from the clustering step through alpha diversity. The linear discriminant analysis, combined with effect size measurements (LEfSe), were used to quantitate differential taxonomic and functional pathway abundance between the groups (R vs. NR).

\section{Statistical analysis}

Statistical analyses were performed using the EZR version 1.30 statistical software (12). All statistical tests were twosided, and $\mathrm{P}<0.05$ was regarded as statistically significant. The demographic characteristics were expressed as frequencies and percentages for the categorical variables and as medians and ranges for the continuous variables. The categorical variables were compared using Fisher's exact test. The TTF and OS were calculated using the Kaplan-Meier method, and the differences were compared using the log-rank test. The alpha diversity metrics and relative abundance of the gut microbiomes were compared by Mann-Whitney tests.

\section{Results}

\section{Patient characteristics}

Six NSCLC patients responded to ICI treatment, while 11 


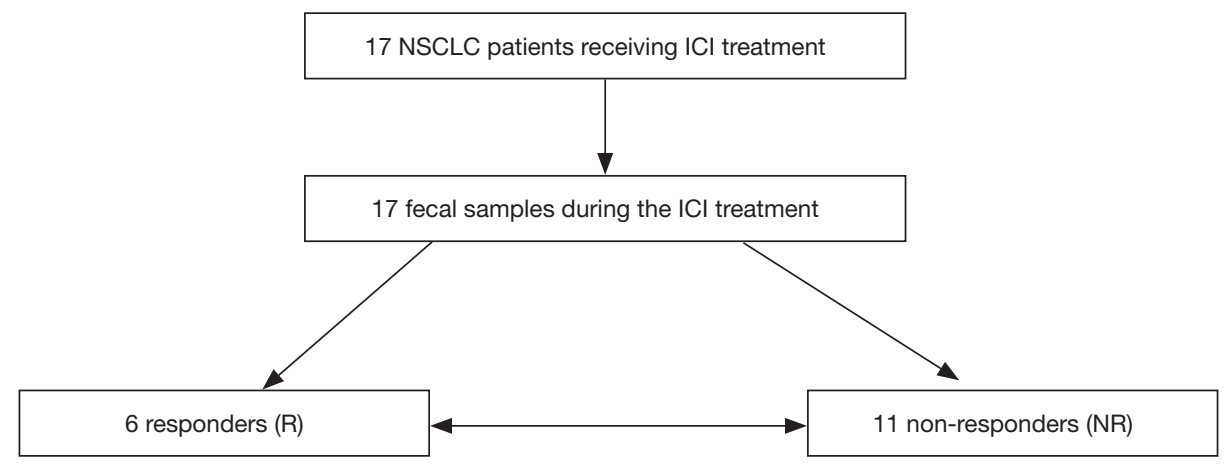

Figure 1 Flowchart of NSCLC patients treated with ICI. A total of 17 NSCLC patients underwent treatment with ICI, and 6 NSCLC patients showed a response to ICI treatment based on the RECIST criteria. NSCLC, non-small cell lung cancer; ICI, immune checkpoint inhibitor.

patients did not respond (Figure 1). A total of 13 (76.5\%) patients were male, and the median age of all patients was 70 years (range, 56-83 years). The performance statuses were 0 in $5(29.4 \%)$, and 1 in $12(70.6 \%)$ patients. No patient experienced a CR (0\%), 6 patients had a PR (35.3\%), 10 had an SD (58.8\%), and 1 had a PD (5.9\%) when treated with ICIs according to the RECIST criteria indicating a response rate of $35.3 \%$. The median TTF was 270 days for all NSCLC patients. OS could not be assessed because most patients were alive at the end of the study. The median follow-up time was 622 days for all NSCLC patients. The baseline clinicopathological characteristics of the patients are summarized in Table 1 .

\section{Association between the gut microbiome and outcome of ICIs in NSCLC patients}

We performed $16 \mathrm{~S}$ rRNA sequencing on the stool samples collected during ICI treatment and evaluated high dimensional class comparisons via LEfSe on the population of gut microbiota between the responders and non-responders of ICIs. The gut microbiomes in the ICI responders were significantly enriched with Lactobacillus, Clostridium, and Syntrophococcus when compared to those of non-responders. In contrast, the gut microbiomes of the ICI non-responders were significantly abundant with Bilophila, Sutterella, and Parabacteroides when compared to those of responders (Figure 2). Patients with a high abundance of Lactobacillus, Clostridium, and Syntrophococcus tended to have a longer TTF when compared to those with a lower abundance. Patients with a low amount of Bilophila and Sutterella had a significantly prolonged TTF than those with a high amount. However, there was no remarkable association between the abundance of Parabacteroides and TTF with ICIs (Figure 3). The alpha-diversity of the gut microbiota was not significantly different between the responders and non-responders and did not influence the TTF (Figure S1).

\section{Discussion}

In this study, we identified that the colonization of the operational taxonomic unit, including Lactobacillus and Clostridium, was associated with favorable clinical outcomes with ICI treatment. Lactobacillus has been reported to promote DC maturation and regulate host immunity in preclinical models (13). These observations suggest that the specific subpopulation enrichment of the gut microbiomes, such as Lactobacillus and Clostridium, may elicit $\mathrm{T}$ cell mobilization to the tumors, thereby enhancing the therapeutic effects of ICI treatment. In contrast, a subpopulation of the microbiome, such as Sutterella and Bilophila, was negatively associated with TTF with ICI treatment. These bacteria elicit inflammation within the tumor microenvironment, which may be related to the insensitivity to ICI treatment $(14,15)$. Interestingly, Bilophila-induced inflammation and intestinal barrier dysfunctions have been shown to be suppressed by the probiotic, Lactobacillus, in mice models (14). Therefore, the management with probiotics may improve the response to maintenance therapy with ICIs. Further investigations are warranted to improve the clinical outcomes of NSCLC patients with ICIs.

This study has several limitations. First, it comprised a small retrospective sample and selected only patients who were undergoing ICI treatment for a long duration. Second, 
Table 1 Patient characteristics at baseline $(n=17)$

\begin{tabular}{|c|c|}
\hline Items & Values \\
\hline Age (years), median [range] & 70 [56-83] \\
\hline \multicolumn{2}{|l|}{ Gender, n (\%) } \\
\hline Male & $13(76.5)$ \\
\hline Female & $4(23.5)$ \\
\hline \multicolumn{2}{|l|}{ ECOG-PS, n (\%) } \\
\hline 0 & $5(29.4)$ \\
\hline 1 & $12(70.6)$ \\
\hline \multicolumn{2}{|l|}{ Histology, n (\%) } \\
\hline Adenocarcinoma & $10(58.8)$ \\
\hline Squamous cell carcinoma & $3(17.6)$ \\
\hline Other & $4(23.5)$ \\
\hline \multicolumn{2}{|l|}{ Smoking status, n (\%) } \\
\hline Never smoker & $4(23.5)$ \\
\hline Current or former smoker & $13(76.5)$ \\
\hline \multicolumn{2}{|l|}{ Staging, n (\%) } \\
\hline Stage IV & $8(47.1)$ \\
\hline Stage III & $6(35.3)$ \\
\hline Postoperative recurrence & $3(17.6)$ \\
\hline \multicolumn{2}{|l|}{ EGFR mutations, $\mathrm{n}(\%)$} \\
\hline Positive & $1(5.9)$ \\
\hline Negative & $16(94.1)$ \\
\hline \multicolumn{2}{|l|}{ PD-L1 TPS, n (\%) } \\
\hline$\geq 50 \%$ & $8(47.1)$ \\
\hline $1-49 \%$ & $4(23.5)$ \\
\hline$<1 \%$ & $1(5.9)$ \\
\hline Not evaluation & $4(23.5)$ \\
\hline \multicolumn{2}{|l|}{$\mathrm{ICl}, \mathrm{n}(\%)$} \\
\hline Nivolumab & $6(35.3)$ \\
\hline Pembrolizumab & $11(64.7)$ \\
\hline \multicolumn{2}{|c|}{ Response to ICI treatment, $\mathrm{n}(\%)$} \\
\hline $\mathrm{CR} / \mathrm{PR}$ & $6(35.3)$ \\
\hline SD & $10(58.8)$ \\
\hline PD & $1(5.9)$ \\
\hline
\end{tabular}

Table 1 (continued)
Table 1 (continued)

\begin{tabular}{lc}
\hline Items & Values \\
\hline TTF of ICIs, $\mathrm{n}(\%)$ & $5(29.4)$ \\
$3-6$ months & $5(29.4)$ \\
$6-12$ months & $7(41.2)$ \\
More than 12 months & \\
Previous chemotherapy regimen, $\mathrm{n}(\%)$ & $12(70.6)$ \\
Platinum regimen & $7(41.2)$ \\
Docetaxel & $4(23.5)$ \\
Others & \\
Previous lines of treatment received, $\mathrm{n}(\%)$ & $4(23.5)$ \\
0 & $10(58.8)$ \\
1-2 & $3(17.6)$ \\
$>3$ & $16(94.1)$ \\
Administration of corticosteroids (30 days \\
before and after beginning ICl), $\mathrm{n}(\%)$
\end{tabular}

$\mathrm{ICI}$, immune checkpoint inhibitor; TTF, time to treatment failure.

we selected patients who were being treated with ICIs for more than three months, which may cause a selection bias. Third, there may be a bias considering that we obtained data from our domestic hospital only. Forth, we evaluated the outcomes only once based on stool sampling during ICI treatment, and we have not evaluated the influence of ICI treatment on the gut microbiome. Therefore, a further prospective study is warranted to identify the role of the microbiome in evaluating the response to ICIs in NSCLC patients.

\section{Conclusions}

In summary, our observations suggest that the composition 
A
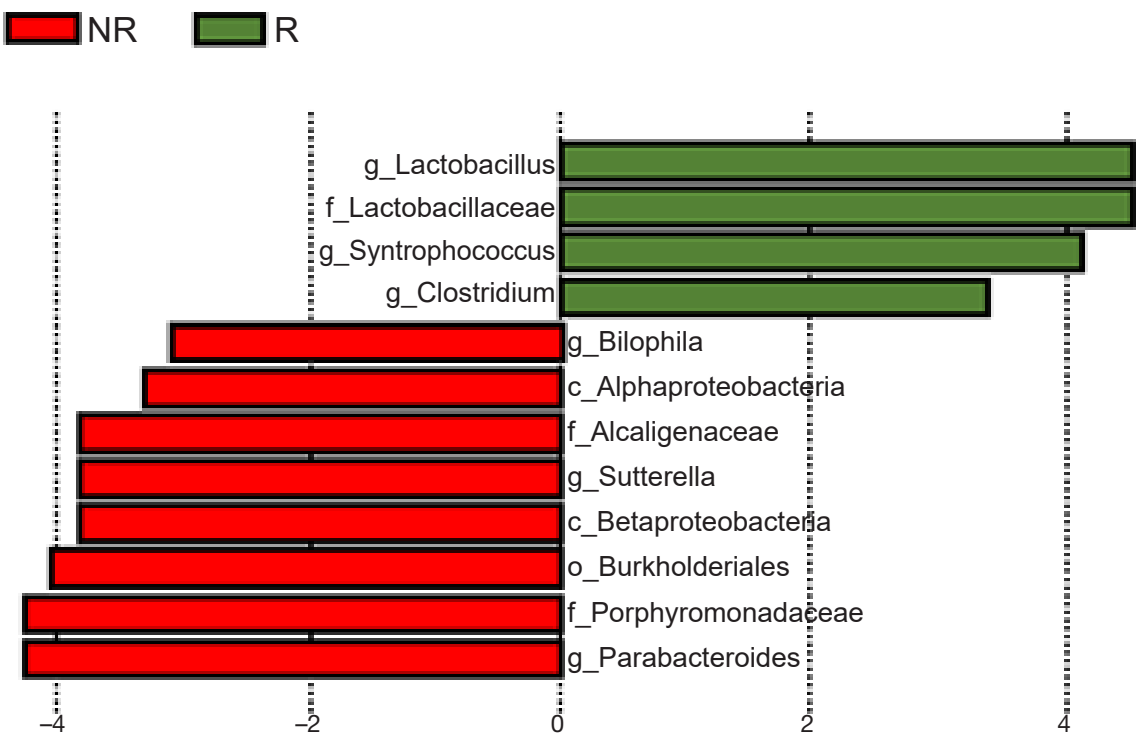

B

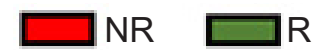

LDA SCORE $(\log 10)$

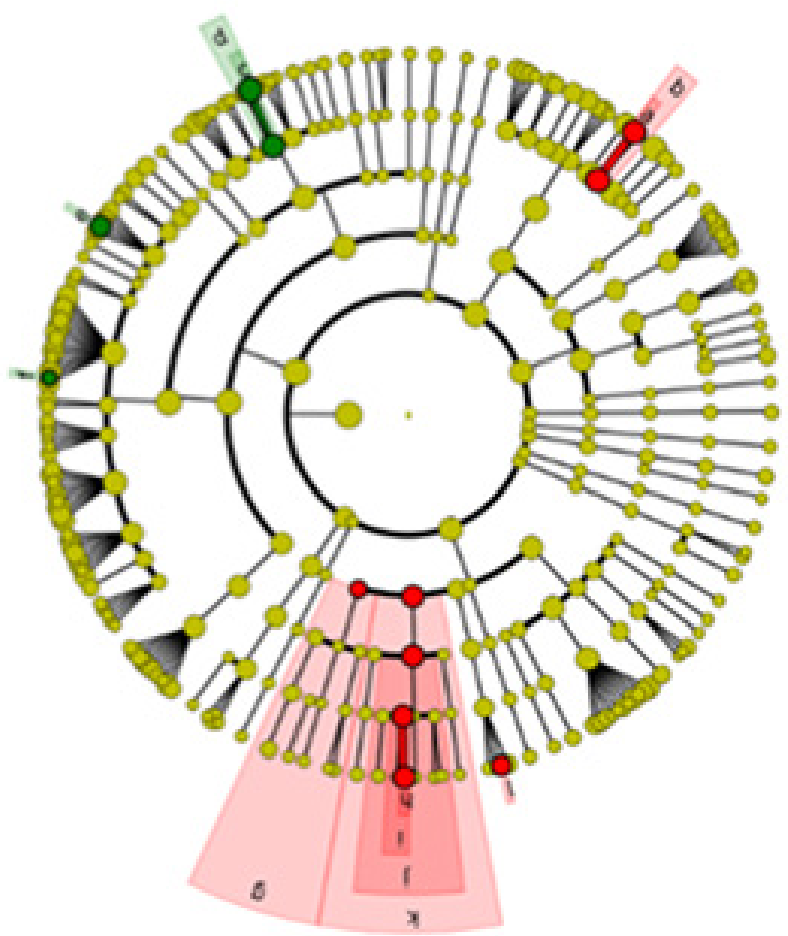

a: g_Parabacteroides

b: f_Porphyromonadaceae

$\square$ c: g_Lactobacillus

d:f_Lactobacillaceae

e: g Clostridium

f: g_Syntrophococcus

$\square$ g: c_Alphaproteobacteria

h: g_Sutterella

$\square$ i: f_Alcaligenaceae

j:o_Burkholderiales

k: c_Betaproteobacteria

I: g_Bilophila

Figure 2 Compositional differences in the gut microbiome. (A,B) The differential abundant taxa in the gut microbiomes of R (green) and NR (red) was analyzed by linear discriminate analysis coupled with effect size measurements (LEfSe) projected with a histogram or cladogram. The length indicates the effect size associated with a taxon. $\mathrm{P}=0.05$ for the Kruskal-Wallis H statistic, LDA score of $>3$. The gut microbiomes in the ICI responders were significantly enriched with Lactobacillus $(\mathrm{P}=0.032)$, Clostridium ( $\mathrm{P}=0.048)$, and Syntrophococcus $(\mathrm{P}=0.0029)$ as compared to those of the non-responders. In contrast, gut microbiomes in the ICI non-responders were significantly abundant with Bilophila $(\mathrm{P}=0.033)$, Sutterella $(\mathrm{P}=0.035)$, and Parabacteroides $(\mathrm{P}=0.027)$ as compared to those in the responders. ICI, immune checkpoint inhibitor. 

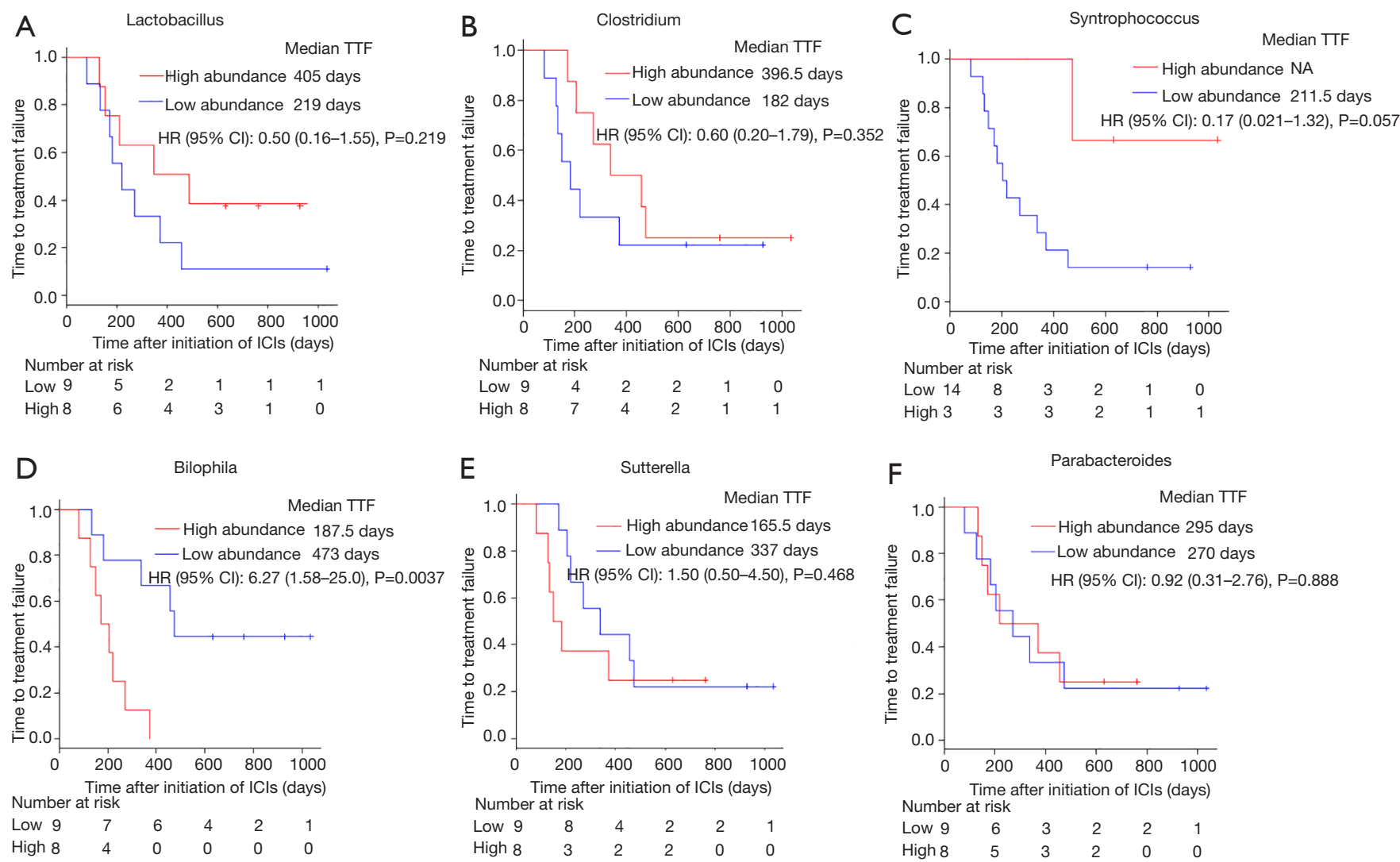

Figure 3 The correlation between the gut microbiome and responses to immunotherapy. The comparison KM plot TTF curves by log-rank test in patients with high abundance (red line) or low abundance (blue line) of Lactobacillus (median TTF: 405 vs. 219 days, P=0.219) (A), Clostridium (median TTF: 396.5 vs. 182 days, $\mathrm{P}=0.352$ ) (B), Syntrophococcus (median TTF: NA vs. 211.5 days, P=0.057) (C), Bilophila (median TTF: 473 vs. 187.5 days, $\mathrm{P}=0.0037)$ (D), Sutterella (median TTF: 337 vs. 165.5 days, $\mathrm{P}=0.468)(\mathrm{E}$ ), and Parabacteroides (median TTF: median TTF: 295 vs. 270 days, $\mathrm{P}=0.888)(\mathrm{F})$.

of the gut microbiome may be associated with therapeutic outcomes in patients with advanced NSCLC treated with ICIs for a long time. A further large-scale study is warranted to validate the composition of the gut microbiome as a novel clinical factor for the response to ICIs in NSCLC patients after a long duration.

\section{Acknowledgments}

Funding: This work was supported by the Kyoto Health Science Research Center (Kyoto, Japan).

\section{Footnote}

Conflicts of Interest: Dr. Yamada reports receiving research grants from Pfizer Inc., Ono Pharmaceutical Co., Ltd., Takeda Pharmaceutical Co. Ltd., and Chugai
Pharmaceutical Co., Ltd. Dr. Uchino reports receiving research grants from Eli Lilly Japan K.K., AstraZeneca K.K., and Boehringer Ingelheim Japan Inc. Dr. Takayama reports receiving research grants from Chugai-Roche Co., and Ono Pharmaceutical Co., and personal fees from AstraZeneca Co., Chugai-Roche Co., MSD-Merck Co., Eli Lilly Co., Boehringer-Ingelheim Co., and Daiichi-Sankyo Co. The other authors have no conflicts of interest to declare.

Ethical Statement: The authors are accountable for all aspects of the work in ensuring that questions related to the accuracy or integrity of any part of the work are appropriately investigated and resolved. The study protocol was approved by the Ethics Committees of Kyoto Prefectural University of Medicine and each hospital, and conducted in accordance with the principles of the Declaration of Helsinki. This was a retrospective study, so 
informed consent was waived.

\section{References}

1. Borghaei H, Paz-Ares L, Horn L, et al. Nivolumab versus Docetaxel in Advanced Nonsquamous Non-Small-Cell Lung Cancer. N Engl J Med 2015;373:1627-39.

2. Brahmer J, Reckamp KL, Baas P, et al. Nivolumab versus Docetaxel in Advanced Squamous-Cell Non-Small-Cell Lung Cancer. N Engl J Med 2015;373:123-35.

3. Herbst RS, Baas P, Kim DW, et al. Pembrolizumab versus docetaxel for previously treated, PD-L1-positive, advanced non-small-cell lung cancer (KEYNOTE-010): a randomised controlled trial. Lancet 2016;387:1540-50.

4. Rittmeyer A, Barlesi F, Waterkamp D, et al. Atezolizumab versus docetaxel in patients with previously treated non-small-cell lung cancer (OAK): a phase 3, openlabel, multicentre randomised controlled trial. Lancet 2017;389:255-65.

5. Sacher AG, Gandhi L. Biomarkers for the Clinical Use of PD-1/PD-L1 Inhibitors in Non-Small-Cell Lung Cancer: A Review. JAMA Oncol 2016;2:1217-22.

6. Belkaid Y, Hand TW. Role of the microbiota in immunity and inflammation. Cell 2014;157:121-41.

7. Ley RE, Peterson DA, Gordon JI. Ecological and evolutionary forces shaping microbial diversity in the human intestine. Cell 2006;124:837-48.

Cite this article as: Katayama Y, Yamada T, Shimamoto T, Iwasaku M, Kaneko Y, Uchino J, Takayama K. The role of the gut microbiome on the efficacy of immune checkpoint inhibitors in Japanese responder patients with advanced nonsmall cell lung cancer. Transl Lung Cancer Res 2019;8(6):847853. doi: $10.21037 /$ tlcr.2019.10.23
8. Gopalakrishnan V, Spencer CN, Nezi L, et al.

Gut microbiome modulates response to anti-PD-1 immunotherapy in melanoma patients. Science 2018;359:97-103.

9. Matson V, Fessler J, Bao R, et al. The commensal microbiome is associated with anti-PD-1 efficacy in metastatic melanoma patients. Science 2018;359:104-8.

10. Jin Y, Dong H, Xia L, et al. The Diversity of Gut Microbiome is Associated With Favorable Responses to Anti-Programmed Death 1 Immunotherapy in Chinese Patients With NSCLC. J Thorac Oncol 2019;14:1378-89.

11. Routy B, Le Chatelier E, Derosa L, et al. Gut microbiome influences efficacy of PD-1-based immunotherapy against epithelial tumors. Science 2018;359:91-7.

12. Kanda Y. Investigation of the freely available easy-touse software 'EZR' for medical statistics. Bone Marrow Transplant 2013;48:452-8.

13. Elawadli I, Brisbin JT, Mallard BA, et al. Differential effects of lactobacilli on activation and maturation of mouse dendritic cells. Benef Microbes 2014;5:323-34.

14. Natividad JM, Lamas B, Pham HP, et al. Bilophila wadsworthia aggravates high fat diet induced metabolic dysfunctions in mice. Nat Commun 2018;9:2802.

15. Hiippala K, Kainulainen V, Kalliomaki M, et al. Mucosal Prevalence and Interactions with the Epithelium Indicate Commensalism of Sutterella spp. Front Microbiol 2016;7:1706. 


\section{Supplementary}

A

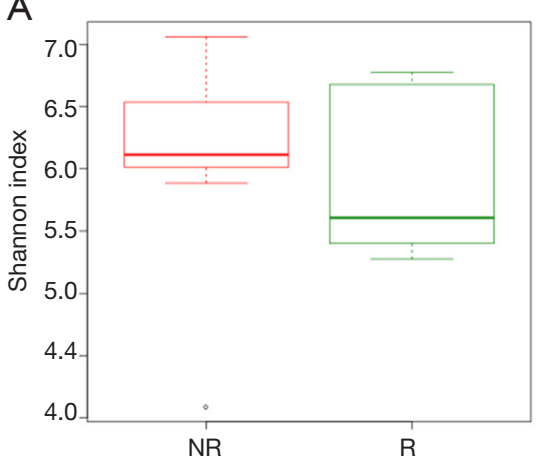

B

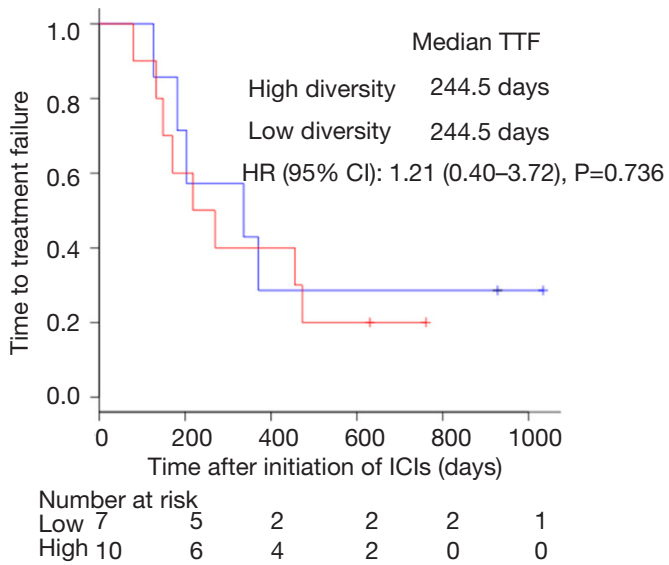

Figure S1 The correlation between the diversity of gut microbiome and responses to immunotherapy. (A) Alpha diversity scores of the gut microbiome (Shannon index) in NR (red) and R (green) by Mann-Whitney (MW) test. (B) The comparison KM plot TTF curves by log-rank test in patients with high diversity (red line) or low diversity (solid line) of the gut microbiome. TTF, time to treatment failure. 